\title{
Gallbladder Nerve Fiber Degeneration: Reactive Drug-induced Change?
}

TO THE EDITOR: Nerve fiber degeneration (NFD) is rarely encountered on abdominal surgical resection specimens. ${ }^{1-3}$ We had recently the opportunity to detect NFD on a gallbladder resection specimen. A multifocal neuroma was detected along with an increased number of normally-sized nerves in the gallbladder adventitia. The medical history revealed a cholecystectomy (20-years previously) as well as chronic rhinitis and rheumatoid arthritis. The treatments included methotrexate, etanercept, tetrazepam, omeprazole, and acetylcysteine.

The multifocal traumatic neuroma consisted in 6 non-encapsulated foci. These foci, measuring 1-4 $\mathrm{mm}$, showed an increased number of nerve fibers, of varied sizes, densely packed (Figure). One of the foci (1-mm) showed 2 reactive lymphocyte nodules.
The largest neuroma-type focus, showed S100- and CD56-positive nerve fibers, disposed concentrically, mixed with epithelial membrane antigen-positive perineural cells. A normal-sized nerve, detected at $1 \mathrm{~mm}$ from this neuroma-focus showed a rounded, giantcell-shaped degeneration zone. This intra-nerve S100- and CD56positive material (and epithelial membrane antigen-negative) contained rare nuclei. There was no inflammatory infiltrate at contact to this nerve.

Here we report intra-nerve NFD in a gallbladder adventitia nerve, at proximity to a multifocal post-traumatic neuroma. The NFD was positive for S100 and CD56 and contained rare nerve fiber nuclei. The origin of intra-nerve NFD was difficult to precise. The hypothesis of post-resection, compensatory-type lesions
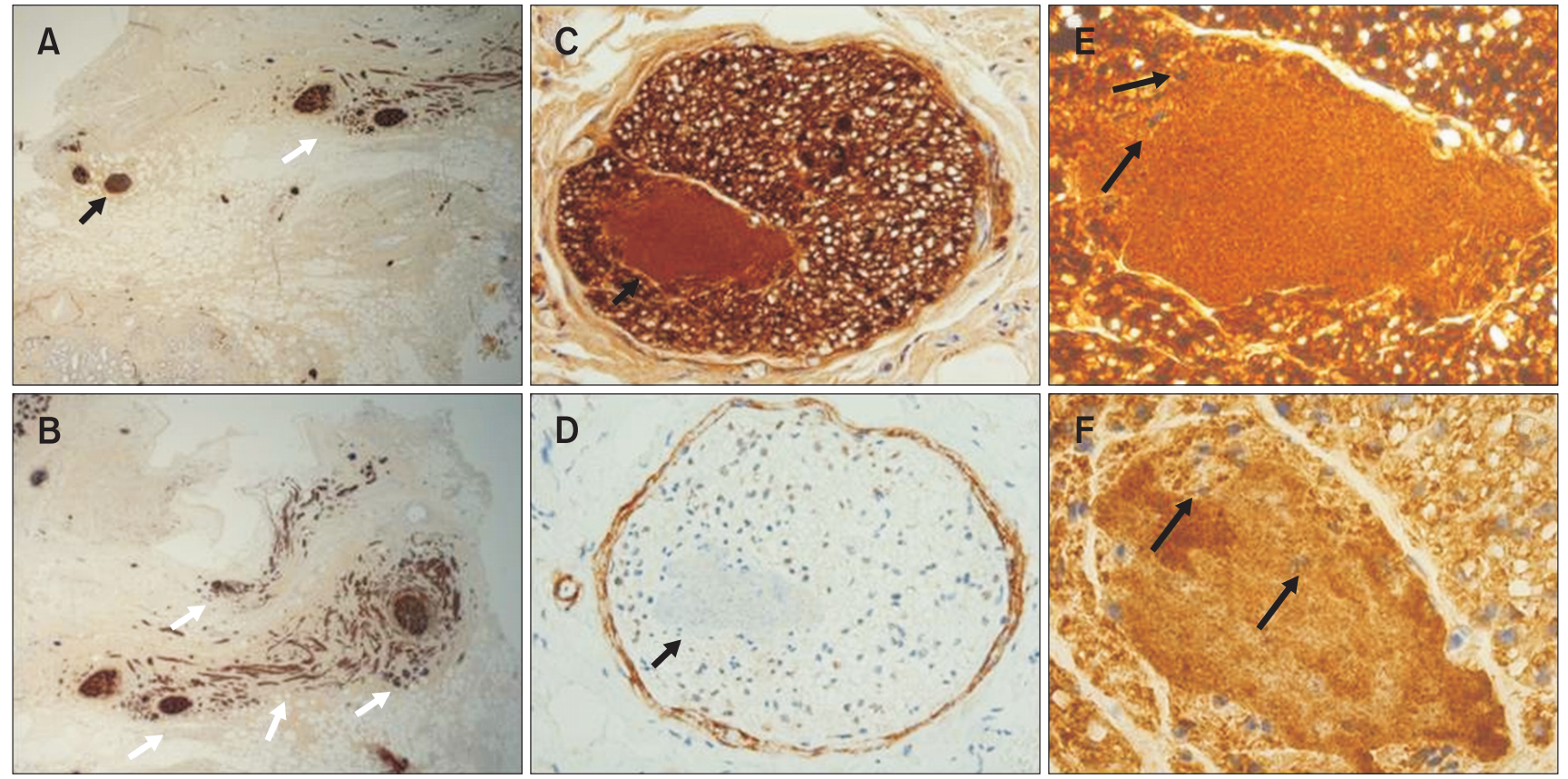

Figure. The traumatic neuroma foci (non-encapsulated, S100-positive) were situated in the gallbladder adventitia at proximity to the muscle layer or close to the serosa or resection limit (A, B: white arrows/neuroma). At proximity of one of the neuroma-foci, there were 2 nerves, one of which contained a "cell-shaped" degeneration zone (A: black arrow; C-F). This lesion was S100-positive (C, E) and CD56-positive (F), and epithelial membrane antigen-negative (D). Rare nerve fiber nuclei were observed in the degeneration zone (E, F: black arrows). Original magnification: $\times 2.5(\mathrm{~A}, \mathrm{~B}), \times 40(\mathrm{C}, \mathrm{D})$, and $\times 100(\mathrm{E}, \mathrm{F})$. 
was difficult to sustain since the initial resection was made 20 -years previously. The hypothesis of degenerative lesions in the context of rheumatoid arthritis was also difficult to sustain as the inflammatory infiltrate almost lacked at contact to the nerve with the intra-nerve degeneration. The 3 rd hypothesis, also difficult to prove, was that of drug/multi-drug-related lesions, pseudo-multiple sclerosis- and/or dysmotility-type reactions being reported for etanercept and tetrazepam. $^{4}$

In conclusion, we report NFD in the context of gallbladder multifocal traumatic neuroma. The pathogenesis of such lesions is difficult to precise whether reactive (compensatory), auto-immune, or drug-related. However, the presence of such lesions should be acknowledged as possibly explaining gallbladder dysmotility.

Adriana Handra-Luca ${ }^{1,2}$

${ }^{1}$ APHP GHU Avicenne, Bobigny, France; and ${ }^{2}$ University Paris Nord, UFR SMBH, Bobigny, France
1. Rosai J. Rosai and Ackerman's surgical pathology: expert consult. 10th ed. Philadelphia: Elsevier 2011.

2. Cabanne F, Bonenfant JL. Anatomie pathologique: principes de pathologie generale, de pathologie speciale et d'etopathologie. 2nd ed. Quebec: Maloine SA 1986.

3. Cotran RS, Kumar V, Collins T. Robbins pathologic basis of disease. 6th ed. Philadelphia: Saunders 1999.

4. Available from URL: https://eurekasante.vidal.fr (accessed 15 Jul 2019).

Acknowledgements: The authors thank Professor Alison Loescher, Ali Khurram, and Armand Adrien as well as the administrative and technical teams of the HUPSSD and the CDMP/APHP and BIUM teams.

Financial support: None.

Conflicts of interest: None. 